\title{
Noncontact Monitoring of Relative Changes in Blood Pressure Using Microwave Radar Sensors
}

\author{
Satoshi Suzuki', Guanghao Sun ${ }^{2}$, Masaaki Hoshiga ${ }^{3}$, Kentaro Kotani' ${ }^{1}$, Takafumi Asao ${ }^{1}$ \\ ${ }^{1}$ Faculty of Engineering Science, Kansai University, Osaka, Japan; ${ }^{2}$ Graduate School of Informatics and Engineering, \\ The University of Electro-Communications, Tokyo, Japan; ${ }^{3}$ Faculty of Medicine, Osaka Medical College, \\ Osaka, Japan
}

Correspondence to: Satoshi Suzuki, ssuzuki@kansai-u.ac.jp

Keywords: Microwave Radar, Noncontact Monitoring, Blood Pressure, Relative Change, Continuous Monitor Received: November 5, $2021 \quad$ Accepted: January 25, $2022 \quad$ Published: January 28, 2022

Copyright $\odot 2022$ by author(s) and Scientific Research Publishing Inc.

This work is licensed under the Creative Commons Attribution International License (CC BY 4.0).

http://creativecommons.org/licenses/by/4.0/

\section{Open Access}

\section{ABSTRACT}

This study aims to confirm whether noncontact monitoring of relative changes in blood pressure can be estimated using microwave radar sensors. First, an equation to estimate blood pressure was derived, after which, the effectiveness of the estimation equation was confirmed using data obtained by a noncontact method while inducing variations in blood pressure. We considered that the Bramwell-Hill equation, which contains some parameters that directly indicate changes in blood pressure, would be an appropriate reference to construct an estimation equation for the noncontact method, because measurements using microwave radar sensors can measure minute scale motion on the skin surface induced by the pulsation of blood vessels. In order to estimate relative changes in blood pressure, we considered a simple equation including the pulse transit time (PTT), amplitude of signals and body dimensions as parameters. To verify the effectiveness of the equation for estimating changes in blood pressure, two experiments were conducted: a cycling task using an ergometer, which induces blood pressure fluctuations because of changes in cardiac output, and a task using the Valsalva maneuver, which induces blood pressure fluctuations because of changes in vascular resistance. The results obtained from the two experiments suggested that the proposed equation using microwave radar sensors can accurately estimate relative changes of blood pressure. In particular, relatively favorable results were obtained for the changes in blood pressure induced by the changes in cardiac volume. Although many issues remain, this method could be expected to contribute to the continuous evaluation of cardiac function while reducing the burden on patients. 


\section{INTRODUCTION}

According to a report by the World Health Organization, ischemic heart disease and stroke are the leading causes of death around the world, accounting for a combined 17.9 million deaths in 2019 [1]. These diseases have remained the leading causes of death globally for the last 15 years. In addition, populations in advanced countries are rapidly aging, which is expected to exacerbate this situation. Therefore, daily monitoring and management in relation to the cardiovascular system are becoming increasingly important to help prevent heart disease.

To manage and prevent heart disease, the most notable parameter that should be monitored is blood pressure. To ensure the quality and accuracy of measurements in hospitals, blood pressure is measured using a catheter inserted into an artery; this is referred to as invasive monitoring. Several other noninvasive methods that enable blood pressure to be easily monitored at home, such as the auscultatory method using Korotkoff sounds, the oscillometric method and the tonometry method [2], have been proposed. The electronic sphygmomanometer used in oscillometric measurements has become popular in home medical care. None of these methods involve invasive measurements, but are premised on using a compression garment, called a manchette or cuff, that applies pressure to the upper arm, wrist, or finger. The physical stress caused by this compression can be a heavy burden on the patient and may be accompanied by several complications, including an increased risk of congestion and undesired side effects for patients with arteriosclerotic vascular disease and arteriosclerotic hypertension [3]. If nighttime blood pressure monitoring is necessary, the sounds of the pumps or servomotors and the physical pressure to the body may interfere with sleep [4]. Moreover, a well-known effect described as "white coat hypertension" [5] often occurs, which refers to the fact that the patient's blood pressure is likely to be higher in a hospital setting than at home. Therefore, it is beneficial for the patient to remain unaware of when his or her blood pressure is being monitored, because it can be highly sensitive to changes in emotional states. Accordingly, blood pressure should be monitored without the patient's knowledge, and preferably, without direct contact. Furthermore, when applying emergency first aid to patients who are in a state of shock, it is important to understand trends in blood pressure fluctuations rather than knowing absolute values. This means that measurements of relative values over time would be appropriate in such situations. The same can be said about the treatment and management of cardiovascular disease.

Various noninvasive sensing techniques have been developed to measure human vital signs. Several noninvasive pulse monitoring studies have been conducted using a strain gauge [6] and piezoelectric sensors [7]. Some research groups have reported a method for measuring a subject's heartbeat and respiration using a multi sensor system [8]. In recent years, novel vital sign monitoring methods using microwave radar sensors have been proposed. Such methods can be used to monitor vital signs without the need for the monitoring devices to come into direct contact with the body. Some reports have demonstrated the practical use of such systems; for example, a microwave radar attached to the ceiling was used to monitor the respiratory rates of subjects in bed through thick bedding $[9,10]$, and another to the back of a chair to monitor changes in autonomic activation [11]. These noncontact and noninvasive sensing techniques measure the extremely small-scale motions that occur on the surface of the skin as a result of cardiac and respiratory activity [12]. Veins, capillaries, and various blood vessels are present near the surface of the skin, and the motion of these vessels changes with variations in blood pressure. Consequently, it is thought that changes in blood pressure induce changes in the motion at the surface of the skin; therefore, the motion at the surface of the skin contains important information about blood pressure. If any information about changes in blood pressure can be extracted from the signals acquired by noncontact methods using microwave radar sensors, blood pressure would be able to be monitored without having to make direct contact with the body. Sensing techniques using microwave radar can be used to detect the movement of a remote object without having to make contact with that object, and microwave radar sensors can be transmitted through clothing and bedding $[9,10]$. Therefore, monitoring methods using microwave radar can be categorized as being fully noncontact and remote. A noncontact sensing method using reflected speckle patterns has also been proposed and it achieves some positive results [13]. However, due to sensing 
by the photonic imaging, it cannot be measured if there is a shield such as clothes. The successful measurement of changes in blood pressure by microwave radar sensors would thus be considered to be extremely useful, because such measurements could be achieved in a noncontact and unconstrained manner. Specifically in regard to blood pressure measurement, a passive cuffless measurement method has been demonstrated [14]; however, no passive noncontact example is available in the existing report. In addition, the microwave radar method used to measure micro-vibrations at the surface of the skin induced by heartbeats can also be used to obtain a series of noncontact unconstrained bio-signal measurements. As the motion at the surface of the skin is thought to vary with blood pressure, the movement of the surface of the skin is also considered to contain cardiovascular information related to changes in blood pressure, although the component of the motion related to blood pressure is expected to be quite small. Moreover, microwave radar sensors using this sensing technique are physically safe and permitted by law for use in electronic devices. This means that vital signs can be monitored continuously; in other words, this sensing technique is suited for monitoring relative changes in blood pressure.

Given this background, this study aims to confirm whether noncontact monitoring of relative changes in blood pressure can be estimated using microwave radar sensors. To achieve this aim, an equation to estimate blood pressure was first derived, and then the effectiveness of the equation was confirmed using data obtained by noncontact monitoring while inducing variations in blood pressure.

\section{THEORETICAL CONSIDERATIONS}

Blood pressure is defined as the pressure applied to the wall of a blood vessel when blood is delivered through the blood vessel [15]. Computationally, it is defined as the product of the volume of blood ejected from the heart (cardiac stroke volume) and the peripheral vascular resistance [15]. However, in actuality, the mechanism governing blood pressure is quite complex because cardiac stroke volume and peripheral vascular resistance are under the control of many regulatory mechanisms. Therefore, it is common to consider the structure of the cardiovascular system by using a conceptual mechanical equivalent model. Several existing conceptual models of the cardiovascular system can be used as guides when considering blood pressure and its variation.

Several effective pulse propagation models have been proposed. The Moens-Korteweg equation [16] describes the relationship between pulse wave velocity (PWV), defined as the propagation speed of the pressure wave produced by cardiac systole, and the elastic modulus of the artery wall. The Bramwell-Hill equation [17] describes the relationship among the PWV, changes in blood pressure, and changes in artery volume.

The Bramwell-Hill equation assumes that minute changes occur in blood vessel volume and tensile stress when blood is ejected from the left ventricle by cardiac systole and the blood pressure in the blood vessel changes. Thus, this equation gives the relationship between PWV and changes in the volume of the blood vessel due to left ventricular ejection, where $h$ and $r$ are the wall thickness and radius of the blood vessel, respectively, $E$ is the elastic modulus of the arterial wall, and $\rho$ is the density of the blood as:

$$
\mathrm{PWV}=\sqrt{\frac{E h}{2 \rho r}}=\sqrt{\frac{V}{\rho \frac{d V}{d p}}}=\sqrt{\frac{V}{\rho} \cdot \frac{d p}{d V}}
$$

where $V$ is the volume elastic modulus of the blood vessel, $p$ is the pressure, and $d V$ and $d p$ are incremental changes in these respective quantities.

The Bramwell-Hill equation is appropriate to estimate blood pressure because it contains a parameter directly indicating the change in the blood pressure. A proportional relationship can be assumed to exist between the diameter of the blood vessel and the motion at the surface of the skin. At the same time, one of the major characteristics of measurements using microwave radar sensors is that they are able to measure the motion at the surface of the skin. This means that the relative change in blood pressure can be measured with microwave radar sensors without the need to make direct contact. When the motion of the 
arterial wall is transmitted to the skin, it is thought that in some cases, the sensitivity of microwave-based monitoring is poor because the motion of the skin is governed by its elasticity. However, in the case of monitoring an artery near the surface of the skin, such as the radial or external carotid artery, it is assumed that the motion of the surface of the skin is similar to the change in the diameter of the artery; this may enable blood pressure to be estimated without contact because information equivalent to changes in the diameter of the artery can be acquired by microwave measurement.

As previously described, in the Bramwell-Hill equation, PWV is determined using elastic volume modules. Solving this equation for the change $d p$ in blood pressure yields:

$$
d p=\rho \mathrm{PWV}^{2} \frac{1}{V} d V
$$

With reference to previous studies using the cardio-ankle vascular index as an indicator of arterial stiffness normally applied in the diagnosis of arteriosclerosis $[18,19]$, the volume elastic modulus $V$ and its change $d V$ can be converted as follows to the base diameter $D$ of the artery and its change $d D$ to express $d p$ in terms of the base diameter:

$$
d p \approx \rho \mathrm{PWV}^{2} \frac{1}{D / 2} d D
$$

Typically, the density $\rho$ of human blood is ranges between 1.043 and $1.060 \mathrm{~g} / \mathrm{mL}$ [20]. It can be assumed that there is individual variability in the density of blood and that it shows long-term changes. However, it can also be assumed that it does not change rapidly over short periods of time, as indicated by Bramwell [17]. Therefore, with blood pressure as the measurement target, the density of blood $\rho$ can be treated as a constant. Similarly, if the same point of the same artery is always targeted, the base diameter $D$ of the artery at the same measurement point does not change during the measurement period, which means that the base diameter $D$ of the artery can be treated as a constant. Therefore, only two variables are non-negligibly involved in governing changes to blood pressure in this estimation method. Following the conceptual definitions described previously, the change $d p$ in blood pressure is a function of two factors, $d D$, which is affected by cardiac stroke volume, and PWV, which is affected by peripheral vascular resistance, as described by:

$$
d p=2 \mathrm{PWV}^{2} d D
$$

If these two parameters could be measured by microwave sensors, it would be possible to estimate blood pressure using a microwave-based noncontact method. Therefore, acquiring data from microwave sensors and inputting them into the equation were considered.

First, it was determined how information regarding PWV could be obtained. Generally, information is acquired using two methods: electrocardiogram (ECG) measurements and pulse wave recording using a finger probe [21]. The timing of ejection from the left ventricle can be estimated from the $\mathrm{R}$ wave of the ECG, and the time at which the pulse ejected from the left ventricle reaches the finger is estimated from the location of the peak of the oscillation in the pulse waves recorded using the finger probe. The phase difference between the two times acquired using these sensing methods is termed the pulse transit time (PTT) [22]. In this study, two microwave sensors were used based on this commonly applied method.

To acquire information about when blood is ejected from the left ventricle, sensor 1 was placed just under a mattress to allow it to be as near to the heart as possible. To acquire information on the periphery of the body, sensor 2 was placed on the left radial styloid process, as when taking the pulse on the wrist by palpation. The phase difference between the two output signals acquired from the measurement points was considered to be the PTT. The transmitted and received microwave radar sensors were not stable, and peaks corresponding to each pulsation in the output signal acquired from the microwave sensor were sometimes unclear. Thus, the phase differential was calculated for each cardiac beat from the cross-correlation between the two sets of data acquired from the sensors with a data length corresponding to a duration of 10 cardiac beats. 
To calculate PWV, the travel distance is needed, in addition to the PTT. The travel distance, defined as the distance from the left ventricle to the left radial styloid process, was calculated and applied in accordance with the human body measurement method [23]. The arm span $S$ and hand length $L$ of each subject were measured (Figure 1(a)), and the travel distance $T D$ was calculated as:

$$
T D=\frac{1}{2} S-L
$$

From this and the PTT, the PWV was calculated as (Figure 1(b)):

$$
d p=2 \mathrm{PWV}^{2} d D=2\left(\frac{T D}{\mathrm{PTT}}\right)^{2} d D=2\left(\frac{\frac{1}{2} S-L}{\mathrm{PTT}}\right)^{2} d D
$$

\section{METHODS}

\subsection{Experimental Setting and Protocols}

To confirm the effectiveness of the proposed equation to estimate changes in blood pressure, two types of experiments depending independently on systolic volume and peripheral vessel parameters were conducted, as blood pressure is defined herein as the product of these two parameters. Blood pressure was first raised in the cycling task with an ergometer (Aerobike ai-ex, Combi Co., Tokyo), which is frequently used in medical and ergonomics research because the stroke volume can be varied by the physical workload. After the ergometer task, the subsequent reduction in blood pressure was monitored.

To verify the effectiveness of the equation in estimating the change in peripheral vessels, the experiment was conducted using the Valsalva maneuver, which is commonly utilized in medical research; this is recognized by the Institute of Electrical and Electronics Engineers (IEEE) as the standard method for checking the quality of cuffless blood pressure monitors.

1) Experiment 1 for observation of changes in blood pressure based on systolic volume was conducted on 10 healthy male volunteer students ranging in age from 21 to 24 years ( $21.70 \pm 0.67$ years). After

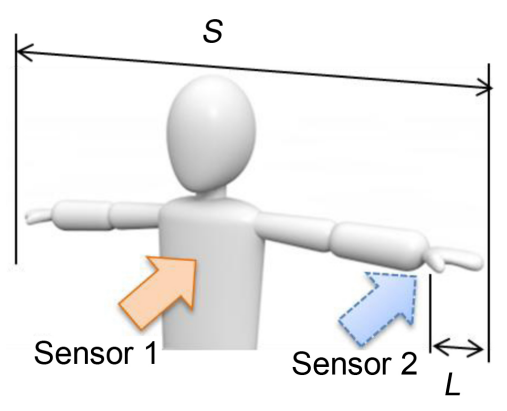

(a)

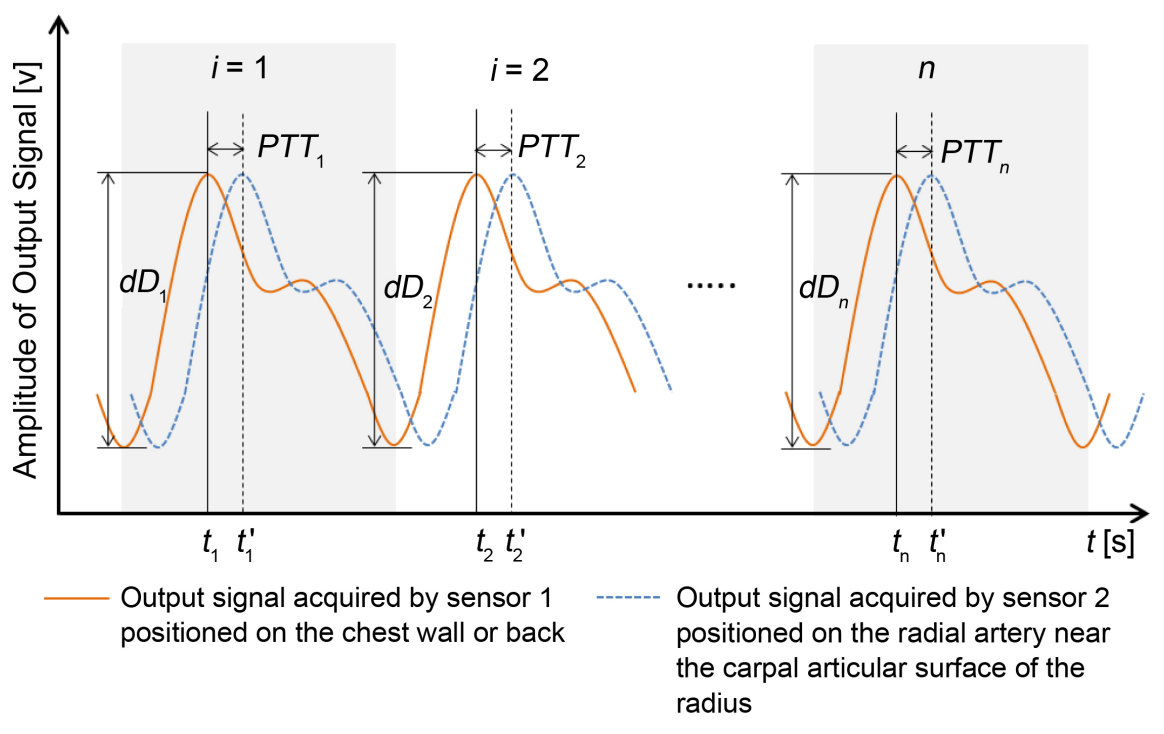

(b)

Figure 1. (a) Measurement points and coordination. (b) Image of acquired waveform and parameters which assigning values to estimation equation. 
informed consent was obtained and instructions regarding the experiment were given, preparations were started. Each healthy volunteer was instructed to lie on a mattress in the supine position with ECG electrodes and pulse wave and blood pressure monitor cuffs in place. Additionally, two microwave sensors were fixed in the mattress $12.5 \mathrm{~mm}$ away from the surface of the back and the inside of the left wrist. After 2 minutes of rest on the mattress, the subject performed the ergometer task for 6 minutes at $150 \mathrm{~W}$ and 60 rpm to raise the blood pressure. After this ergometer task, the subject rested on the mattress again, and blood pressure was monitored for 15 minutes.

2) Experiment 2 for observation of changes in blood pressure based on peripheral vessels was conducted on 10 healthy male volunteer students ranging in age from 21 to 24 years $(21.70 \pm 0.82$ years). After informed consent was obtained and instructions regarding the experiment were given, electrodes and cuffs were placed on the subject's body as described in the previous experiment. The subject was then instructed to lie on a mattress in the supine position and rest for 2 minutes, and the Valsalva maneuver [24] was performed. The subject first inhaled deeply and held his breath. Next, the subject bore down and strained his abdominal muscles for approximately 20 seconds. After 20 seconds, the subject released his breath and resumed normal breathing. The subject then rested for approximately 1 minute. During this test, changes in the ECG measurements, pulse waves, and blood pressure were monitored for reference, and the subject was monitored by two microwave radar sensors that had been installed in the mattress.

The 10 healthy male students selected for the two experiments were in different groups. However, no statistically significant differences in age (Experiment 1: $21.70 \pm 0.67$ years, Experiment 2: $21.70 \pm 0.82$ years, $p=1$, n.s.) or physical characteristics such as height (Experiment 1: $173.6 \pm 5.54 \mathrm{~cm}$, Experiment 2: $169.7 \pm 7.69 \mathrm{~cm}, p=0.11$, n.s.) and weight (Experiment 1: $73.53 \pm 12.56 \mathrm{~kg}$, Experiment 2: $62.90 \pm 8.91 \mathrm{~kg}$, $p=0.12$, n.s.) were observed.

\subsection{Data Acquisition and Analytical Protocols}

The devices used in the two experiments were basically the same. For reference, the subject's precordial ECG was derived with electrodes (Biotop; NEC Sanei Co., Tokyo) placed in the V5 position for monitoring. Additionally, the pulse wave was measured using a finger probe (OLV-3100; Nihon Koden Co., Tokyo) placed on the middle finger of the left hand. Blood pressure in Experiment 1 was monitored every minute for 15 minutes using a manchette on the right arm (BP-1101; Nippon Colin Co., Tokyo). By contrast, in Experiment 2, blood pressure was monitored beat-to-beat using a finger pressure monitor (Finometer MIDI; Finapres Medical Systems BV, Enschede) because blood pressure changes every beat during the Valsalva maneuver (Figure 2(a)). The small cuff of the finger pressure monitor was set on the middle finger of the right hand in Experiment 2 (Figure 2(b)).

Two microwave radar sensors (NJR4261J, New Japan Radio Co., Ltd., Tokyo) were used to acquire cardiac information. To avoid cross talk between the microwave radar sensors, the sensors have two different central frequencies $(24.15$ and $24.11 \mathrm{GHz})$. Each sensor also has a normal output power of $7 \mathrm{~mW}$ (maximum: $10 \mathrm{~mW}$ ) and an antenna gain of $10 \mathrm{dBi}$ with a diffusion angle of approximately $40^{\circ}$ (TS- 01 , New Japan Radio Co., Ltd., Tokyo). To capture the heartbeat when blood was ejected from the left ventricle and to capture the pulsation when the blood reached the radial artery, these two microwave sensors were installed in the mattress positioned $12.5 \mathrm{~mm}$ away from the surface of the back (sensor 1) and the inside of the left wrist (sensor 2). The signals output from the two microwave radar sensors were amplified fiftyfold by a direct current amplifier (DA-710A; Kyowa Co., Tokyo). The output signals from the reference devices and two microwave radar sensors were sampled by an analog-to-digital (A/D) converter (USB-6211; National Instruments Co., Texas) with a sampling interval of 1 millisecond and stored in a personal computer. After sampling with the A/D converter, bandpass filters with a passband of $0.5-2.5 \mathrm{~Hz}$ were applied to both radar outputs to reduce the noise and interference. These bandpass filters cover ranges of 30 - 150 heartbeats per minute. After bandpass filtering, to estimate the change in blood pressure, parameters were acquired from signal data, and the change in blood pressure was estimated using the previously described equation with the acquired parameters as inputs. 

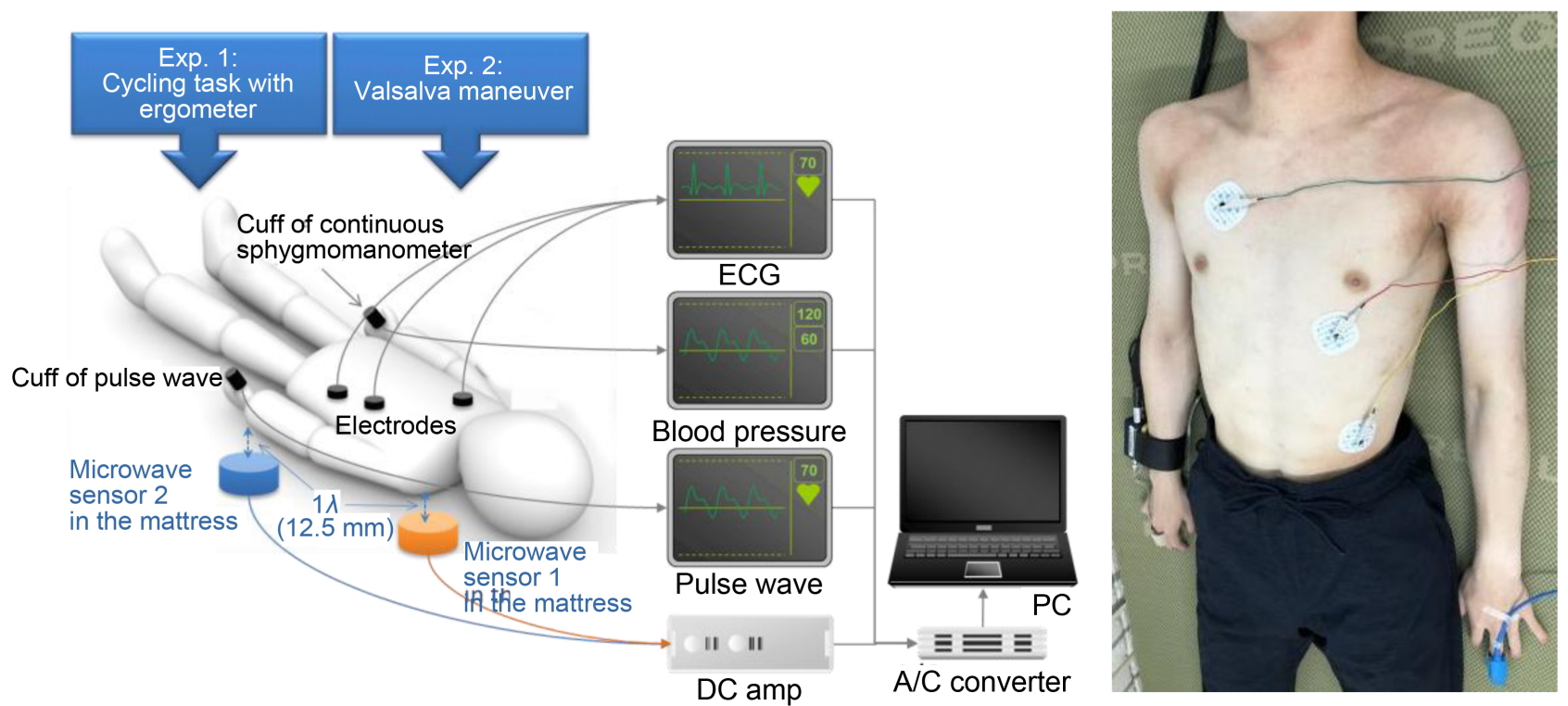

Figure 2. Experimental setting and block diagram of the experiments. (a) Schematic block diagram of the experimental setting. (b) Photograph showing Experiment 2.

Statistical analysis was performed using Excel and non-contact method was evaluated by the correlation coefficient value and the Bland-Altman analysis.

The protocol of this study was reviewed and approved by the Committee on Human Research of Kansai University.

\section{RESULTS}

In Experiment 1, a large overshoot was mixed in the acquired signal data in some subjects because of breathing and body movement, so these data were excluded as abnormal values. Overall, $11.82 \%$ of the values were excluded as abnormal in Experiment 1. On the other hand, in Experiment 2, only 0.77\% of the values were abnormal. However, when the Valsalva maneuver was performed, two of the 10 subjects did not show the common fluctuations indicated in the previous study, and hardly any change in blood pressure was seen. Therefore, the applied load in the Valsalva maneuver was considered insufficient for these two subjects, so the analysis was performed with eight subjects.

Figure 3(a) shows the sample ECG signal of subject $S_{1}$ during 5 seconds in rest period. Figure 3(b) shows the signals corresponding to the cardiac oscillation measured at the same time by the microwave sensors in the mattress capturing the heartbeat from the back and left wrist.

As can be seen in these figures, the output signals measured by the microwave sensors exhibited cyclic oscillations with cardiac motion corresponding to the R-R intervals of the ECG. In addition, the phase shifts between the waveforms obtained by noncontact measurement from the back and radius could be confirmed, indicating the PTT.

Figure 4(a) shows the results of the change in pulse pressure in subject $S_{l}$ in Experiment 1. The horizontal axis represents time and the vertical axis represents changes in pulse pressure. The change in the round marker connected to the dotted line shows the results of the pulse pressure measurement by continuous sphygmomanometer as a reference for the contact measurement method. The change in the diamond-shaped markers connected by the solid line shows the results for the estimated pulse pressure using the estimation equation based on the data acquired by the microwave sensors as noncontact measurement. To compare the degree of change, the results of both the reference and estimated values were normalized. The presented section in Figure 4(a) shows the change during 1000 seconds in the blood pressure 


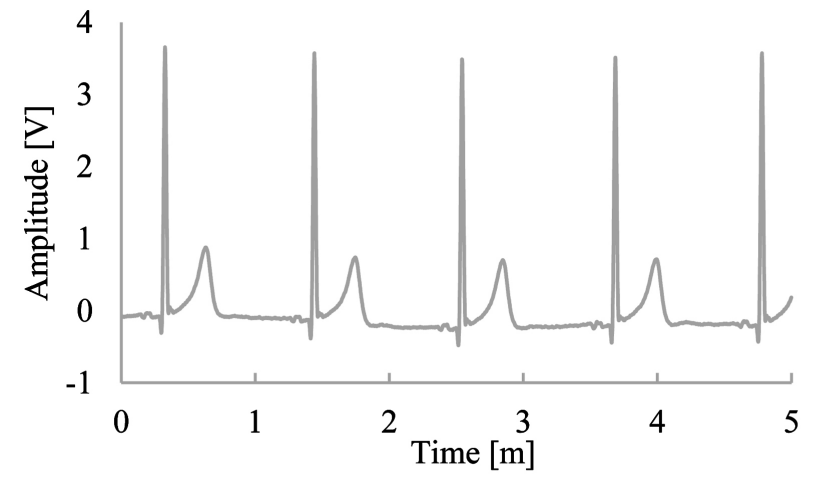

(a)

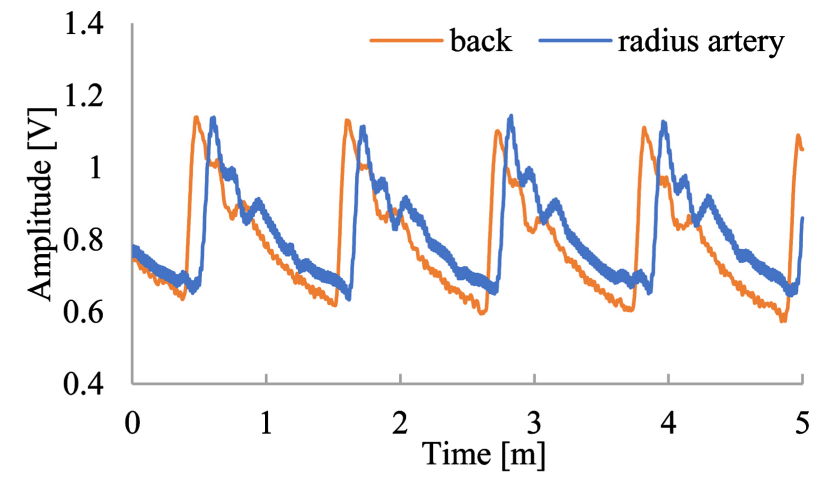

(b)

Figure 3. Sample signals measured by contact and noncontact methods. (a) Sample ECG signal as reference. (b) Sample signals of the noncontact method using microwave radar (upper part acquired at the back and lower part acquired at the radius).

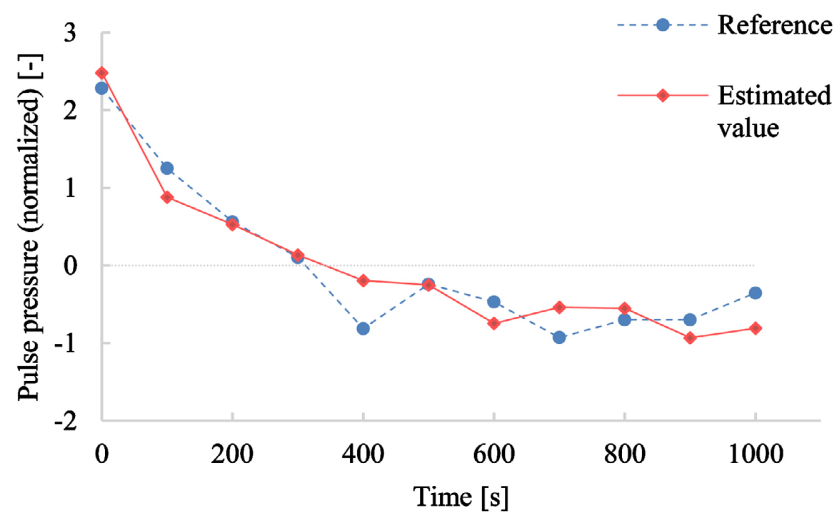

(a)

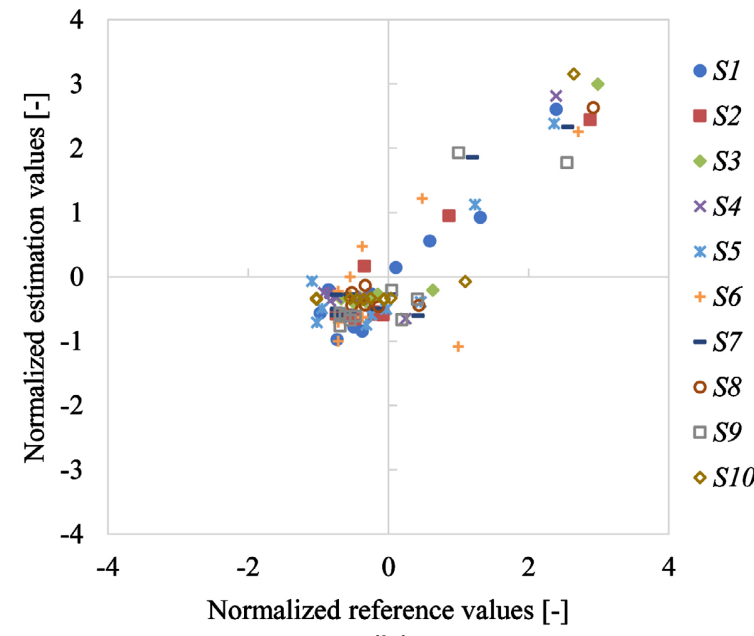

(b)

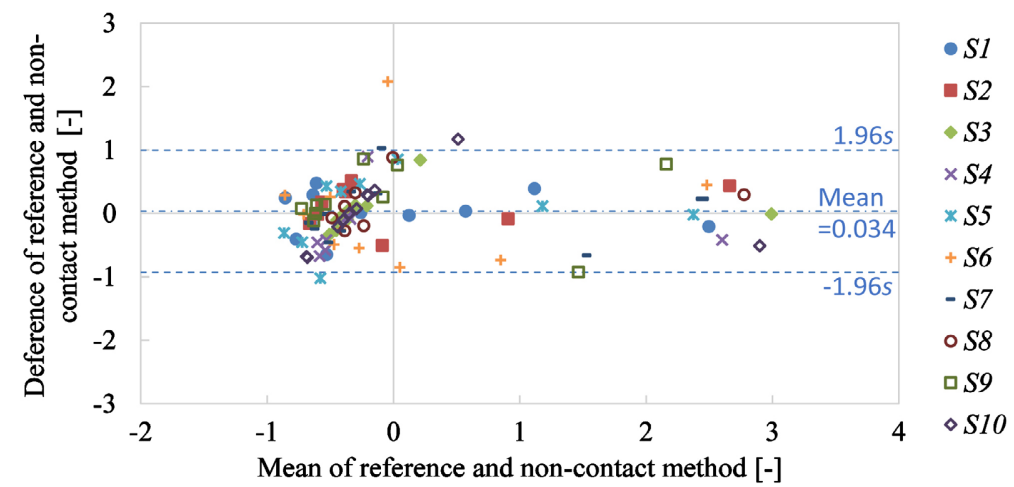

(c)

Figure 4. Results of Experiment 1. (a) Sample of the results of the change in pulse pressure induced by the ergometer task in Experiment 1 (subject $S_{1}$ ). (b) Comparison of the two results measured by the contact method as a reference and the estimation results based on noncontact measurement ( $r=$ 0.884). (c) Bland-Altman plot for all subjects (mean $=0.034$, upper coefficient limit $=10.998$, lower coefficient limit $=-0.930$ ). 
decompressing process from the start of measurement until just after the end of the loading task using the ergometer.

First, from this figure, it can be clearly confirmed that the blood pressure was gradually reduced in a downward trend after the increase due to the physical load in the task using the ergometer. This trend was confirmed in all subjects; it took about 10 minutes for the pulse pressure to decrease to the baseline level. Although the comparison is only of fluctuations because all of the values for both the contact and non-contact measurements are normalized; however, a high correlation coefficient was confirmed and no significant differences were found between the two distributions in this subject $(r=0.946, p=0.500)$. This trend of changes in the two values was very similar, and this tendency was confirmed in all subjects.

Next, Figure 4(b) shows a comparison of the two results measured by the contact method as a reference and the estimation results based on the noncontact measurement in all subjects. Due to the characteristics of this experiment, in which the reduction in blood pressure after applying the physical load was observed, the results are concentrated in the second quadrant immediately after the physical load and concentrated near 0 at rest. However, a high correlation coefficient was confirmed, and no significant difference was found between the two results $(r=0.884, p=0.247)$. Since the correlation coefficient was higher than 0.8 , except for in one subject, the trend of change estimated by the noncontact method was considered to correspond to the reference. Figure 4(c) shows Bland-Altman plots for all subjects (mean = 0.034 , upper coefficient limit $[\mathrm{UCL}]=0.998$, lower coefficient limit $[\mathrm{LCL}]=-0.930$ ). Although the plots are biased to the positive direction because of the characteristics of Experiment 1, they are concentrated in the center of the UCL and LCL, which means that there is no difference between the two methods.

Figure 5 shows the results of Experiment 2. Since there was no change in pulse pressure at the 1-minute rest interval from the start of the experiment, Figure 5(a) shows the results from 10 seconds before the start of the Valsalva maneuver, as well as a total of about 40 seconds during the 15-second Valsalva maneuver and the subsequent 15 seconds recovering to baseline.

The pulse pressure of the contact measurement as the reference was confirmed to decrease from 60 to 75 seconds during loading because of the Valsalva effect. After the load was released, the pulse pressure recovered to the baseline level. This fluctuation is also consistent with the tendency of fluctuations in pulse pressure reported in a previous study $[24,25]$. Additionally, this same tendency was confirmed in both the contact and noncontact measurements. In subject $S_{1}$, a relatively high coefficient correlation was confirmed, and no significant difference was found between the two measurement methods $(r=0.846, p=$ 0.311). Similar changes were confirmed in all eight subjects examined in this experiment, albeit with some individual differences.

As in the case of Figure 4(b), Figure 5(b) shows a comparison of the estimation results by the contact and noncontact measurements in each trial and each subject. From these results, although a certain degree of correlation could be confirmed $(r=0.575, p=0.226)$, the correlation was not as high as that in Experiment 1. Figure 4(c) shows t Bland-Altman plots for all subjects (mean $=0.066, \mathrm{UCL}=1.902, \mathrm{LCL}=$ -1.770). Compared with the results of Experiment 1, variations in the differences between the two methods could be confirmed. The same tendency as that in Figure 5(b) could be confirmed in Figure 5(c).

\section{DISCUSSION}

Comparing the waveforms acquired by contact measurement as a reference and noncontact measurement in Figure 3, it is notable that the same information could be obtained by noncontact sensing because similar characteristics in the cyclic oscillations in signals acquired by the two methods were observed. Previous study provided a graphical model approach to signal modeling using the mechanical movements of the skin relating to the cardiac cycle [25]. In fact, the movements of the skin have some information related to changes of blood pressure. Additionally, since some previous studies have also reported the effectiveness of noncontact measurement using microwave radar sensors [9-11], this noncontact method using microwave radar sensors is considered sufficient for sensing vital signs. Although it is necessary to apply signal processing to remove artifacts and noise, there are some cases in previous reports 


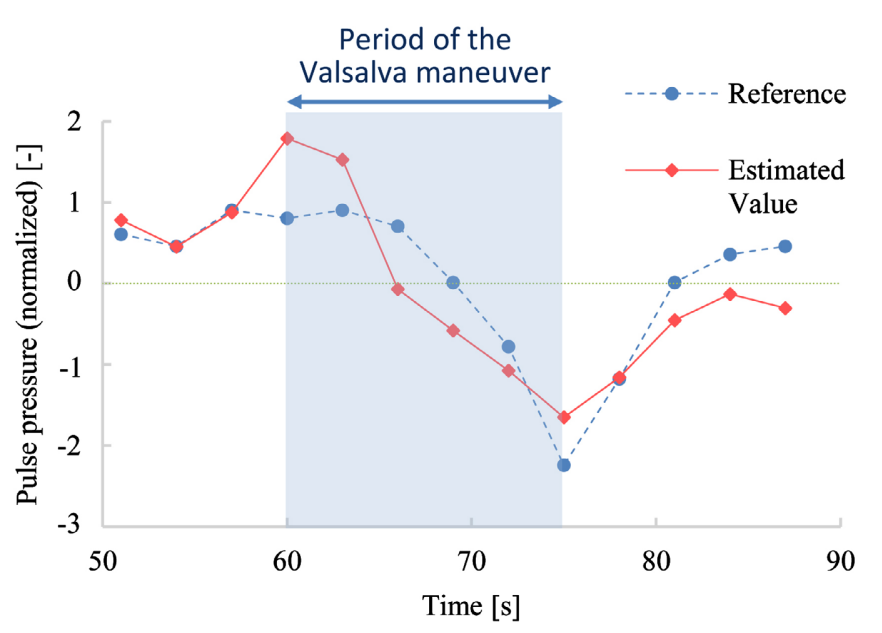

(a)

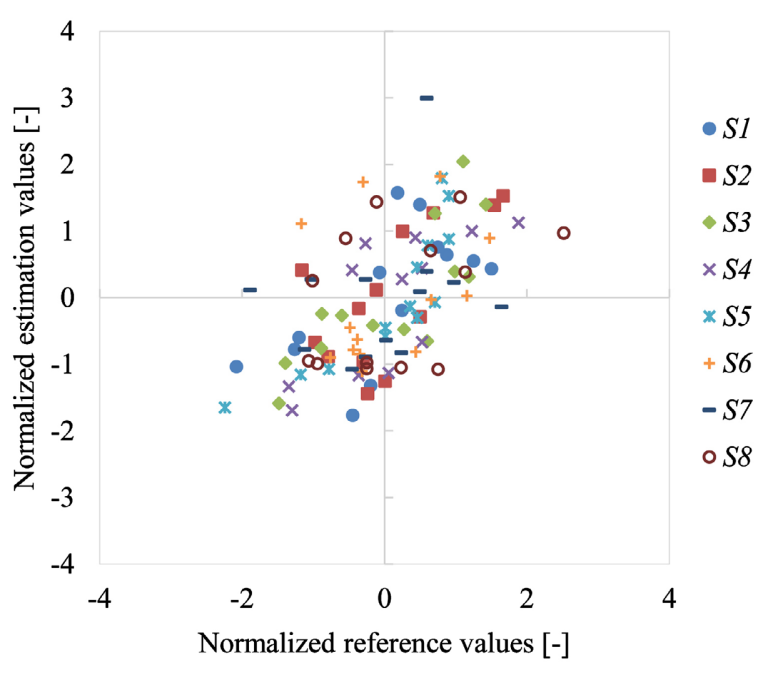

(b)

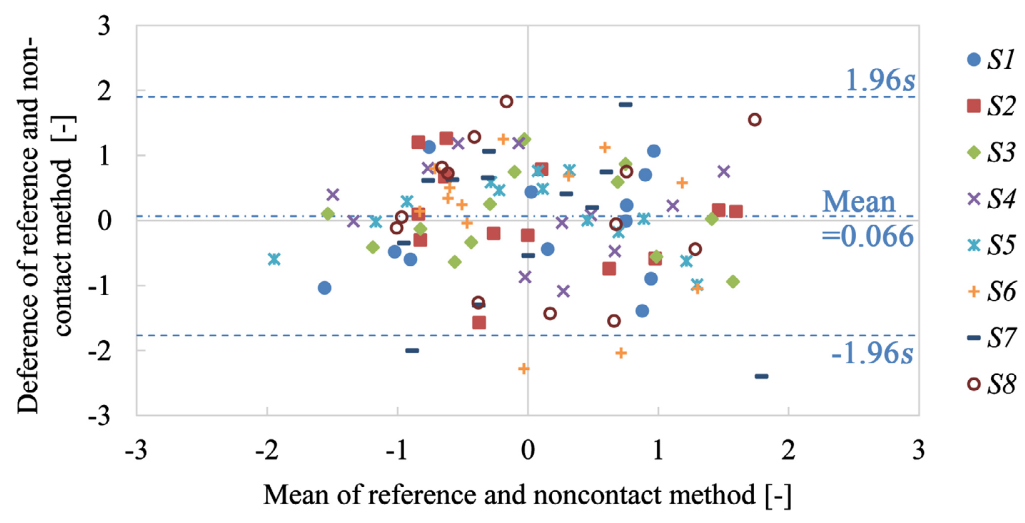

(c)

Figure 5. Results of Experiment 2. (a) Sample of the results of the change in pulse pressure induced by the Valsalva maneuver in Experiment 2 (subject $S_{1}$ ). (b) Comparison of the two results measured by the contact method as a reference and the estimation results based on noncontact measurement $(r=0.575)$. (c) Bland-Altman plots for all subjects (mean $=0.066$, upper coefficient limit $=1.902$, lower coefficient limit $=-1.770)$.

where measurements were taken from 1 meter or more, suggesting the possibility of extracting estimation parameters remotely from a distance farther than that in the present experiment.

Next, as shown in Figure 4 and Figure 5, we compared the estimation results of blood pressure fluctuation measured by the continuous sphygmomanometer as a reference and the noncontact measurement using the estimation equation proposed in this study. As a result, as shown in Figure 4, it was confirmed that the proposed method could estimate the decreasing process of the pulse pressure after blood pressure elevation. This result indicates that the present estimation method can confirm the change in pulse pressure when a relatively large fluctuation occurs because of a change in cardiac output. This clearly suggests that, at the very least, the lowering of blood pressure can be estimated.

On the other hand, although the fluctuation induced by the Valsalva effect in Experiment 2, namely the fluctuation of pulse pressure caused by the change in vascular resistance, was confirmed to some extent, the accuracy was lower than that in Experiment 1. There are several possible causes for this, including the degree of load in Experiment 2 and the response to vascular resistance due to this load, the subjects' personal characteristics, the sensing accuracy, and problems with the estimation equation. 
A likely assumption is that the signal measured using the noncontact method is affected by artifacts or noise. However, compared with Experiment 1, it is unlikely that the influence by noise had an effect. In fact, in Experiment 1, some large overshoots induced by breathing and body movement were included, so some data were excluded as abnormal values. The ratio excluded as abnormal values was about $11.82 \%$, but in Experiment 2, this ratio was $0.77 \%$, which means that the data were largely unaffected by noise or artifacts.

In addition, the fluctuation in blood pressure caused by the change in cardiac output was large, whereas the change due to peripheral vascular resistance was relatively small. Therefore, it can be expected that the result was originally difficult to observe. Actually, in Experiment 1, the mean pulse pressure of all subjects before the task was $59.9 \mathrm{mmHg}(59.90 \pm 7.67 \mathrm{mmHg})$, and increased to $91.4 \mathrm{mmHg}(91.40 \pm 11.34$ $\mathrm{mmHg}$ ) immediately after the task, which represents a change of about $53.0 \%$. On the other hand, regarding the Valsalva maneuver in Experiment 2, the mean pulse pressure of all subjects at rest before the task was $57.19 \mathrm{mmHg}(57.19 \pm 12.79 \mathrm{mmHg})$, but was $58.51 \mathrm{mmHg}(58.51 \pm 14.15 \mathrm{mmHg})$ under the task load of the Valsalva maneuver, which is an extremely small change (2.29\%). Since individual differences also have a large effect, it is considered difficult to obtain a high correlation value and positive results as a whole. Due to the combination of these causes, it is probable that the clear validity of the estimation could not be confirmed.

Although all subjects practiced the Valsalva maneuver multiple times before the experiment, the fluctuations in the reference pulse pressure induced by the Valsalva maneuver in two subjects were different from the general fluctuations reported in previous studies [26, 27]. We judged that the load of the Valsalva maneuver was not sufficiently applied for these two subjects, so we excluded them from the analysis. From this situation, it is considered that the Valsalva maneuver is difficult for subjects to perform as a task load, and that the control is also insufficient.

In medical experiments intended to confirm changes in blood pressure, confirmation is achieved by drug control [28, 29]; however, in this study, the verification was conducted using the task load of the Valsalva maneuver as a noninvasive method normally used for research regarding blood pressure [30, 31]. The actual degree of blood vessel hardening cannot be measured except for the invasive contact method. Therefore, further studies are needed to confirm in detail the blood pressure fluctuations caused by changes in vascular resistance.

Mukkamala et al. [32] conducted a detailed study on a blood pressure estimation equation. They theoretically examined the elastic and viscosity models from the viewpoint of the influence on the mechanical properties of aging and diseases. They concluded that it does not significantly affect the viscous effect and elastic properties due to aging as a theoretical model. However, this remains controversial, as various disease factors are expected to be involved. Thus, it is predicted that estimation by the PTT is effective. Compared with their report, the estimation equation proposed in the present paper also uses two parameters, one related to cardiac volume and the other related to changes in vascular resistance. In addition, although there are differences in the denominator and numerator, the order is the same in that the square of the PWV is used. Thus, the estimation equation proposed in this study is also considered to be valid.

On the other hand, due to the structure of the present estimation equation, the PTT and amplitude intensity exert a strong influence. However, since the results of Experiment 2 were inferior to those of Experiment 1 , there may be a problem with the detection accuracy of the PTT using the microwave radar sensor, or the change in the PTT could not work well in the equation because it was relatively small with respect to the change in $d D$.

Other parameters that may affect accuracy include the subjects' physical characteristics, such as skin conditions. Although some survey results have suggested actual effects [33,34], it is necessary to examine how these parameters will be considered in estimations in the future.

\section{CONCLUSION}

Even though blood pressure is a useful and efficient vital sign for medical diagnoses, blood pressure is 
one of the most difficult vital signs to monitor by noncontact methods, because some parameters must be confirmed with contact such as the density of the blood and elasticity of the blood vessels. Among the various vital signs, blood pressure is the only one for which accurate and completely noncontact monitoring remains elusive.

The purpose of this study was to confirm the possibility estimating relative changes in blood pressure by noncontact monitoring using microwave radar sensors. Especially, based on Bramwell-Hill modeling, we considered and investigated a noncontact measurement method to monitor continuously the relative changes rather than the absolute value of blood pressure. As a result, we confirmed some effectiveness for this method and were able to extract some issues that need to be addressed for application in the future. In particular, the results suggested that it may be possible to estimate the fluctuations in blood pressure induced by changes in cardiac output. On the other hand, although it was also suggested that the fluctuations in blood pressure induced by organic factors in blood vessels could be estimated, it was confirmed that the accuracy was not sufficient; thus, future studies are required. It cannot be denied that many hurdles remain for clinical use, such as considering physical characteristics and improving accuracy, noise processing, and the estimation equation.

However, the present findings can be considered an achievement, because the relative fluctuations in blood pressure could be estimated by a noncontact sensing method, as the correlation coefficient between the actual and noncontact measurements was confirmed. This is considered to be of great significance not only for reducing the burden on patients, but also in clinical diagnoses. In particular, some diseases, such as hypovolemic shock and cardiogenic shock, show fluctuations in blood pressure derived from changes in cardiac output $[35,36]$. Among several criteria in the diagnosis of fluctuations in blood pressure, there is a diagnostic criterion stating that systolic blood pressure is $30 \mathrm{mmHg}$ or more lower than the baseline of blood pressure [37]. It is thought that detection at this level will be possible with the present method, and thus, it is considered to have potential for future applications.

As mentioned above, many countries are facing a rapidly aging population and increasing risks in postoperative management, because older patients normally have multiple underlying diseases. It is thought that the proposed method could provide a diagnostic and management technology for the cardiovascular system in super-aging societies in the future.

\section{ACKNOWLEDGEMENTS}

This work was supported by JSPS KAKENHI Grant No. 19K12884.

\section{CONFLICTS OF INTEREST}

The authors declare no conflicts of interest regarding the publication of this paper.

\section{REFERENCES}

1. World Health Organization (2021) Cardiovascular Diseases (CVDs) Key Facts. https://www.who.int/news-room/fact-sheets/detail/cardiovascular-diseases-(cvds)

2. Ogedegbe, G. and Pickering, T. (2010) Principles and Techniques of Blood Pressure Measurement. Cardiology Clinics, 28, 571-586. https://doi.org/10.1016/j.ccl.2010.07.006

3. Bottini, P.B., Rhoades, R.B., Carr, A.A. and Prisant, L.M. (1991) Mechanical Trauma and Acute Neuralgia Associated with Automated Ambulatory Blood Pressure Monitoring. American Journal of Hypertension, 4, 288. https://doi.org/10.1093/ajh/4.3.288

4. Heude, E., Bourgin, P., Feigel, P. and Escourrou, P. (1996) Ambulatory Monitoring of Blood Pressure Disturbs Sleep and Raises Systolic Pressure at Night in Patients Suspected of Suffering from Sleep-Disordered Breathing. Clinical Science, 91, 45-50. https://doi.org/10.1042/cs0910045 
5. Pickering, T.G., Hall, J.E., Appel, L.J., Falkner, B.E., Graves, J., Hill, M.N., Jones, D.W., Kurtz, T., Sheps, S.G. and Roccella, E.J. (2005) Recommendations for Blood Pressure Measurement in Humans and Experimental Animals: Part 1: Blood Pressure Measurement in Humans-A Statement for Professionals from the Subcommittee of Professional and Public Education of the American Heart Association Council on High Blood Pressure Research. Circulation, 111, 697-716. https://doi.org/10.1161/01.CIR.0000154900.76284.F6

6. Feliciani, G., Peron, C., La Rocca, A., Scuppa, M.F., Malavolta, A., Bianchini, D., Corazza, I. and Zannoli, R. (2016) Cold Pressor Test Using Strain-Gauge Plethysmography. Advances in Physiology Education, 40, 410-417. https://doi.org/10.1152/advan.00096.2015

7. Wang, T.W. and Lin, S.F. (2020) Wearable Piezoelectric-Based System for continuous Beat-to-Beat Blood Pressure Measurement. Sensors (Switzerland), 20, 1-12. https://doi.org/10.3390/s20030851

8. Fajkus, M., Nedoma, J., Martinek, R., Vasinek, V., Nazeran, H. and Siska, P. (2017) A Non-Invasive Multichannel Hybrid Fiber-Optic Sensor System for Vital Sign Monitoring. Sensors (Switzerland), 17, 1-17. https://doi.org/10.3390/s17010111

9. Uenoyama, M., Matsui, T., Yamada, K., Suzuki, S., Takase, B., Suzuki, S., Ishihara, M. and Kawakami, M. (2006) Non-Contact Respiratory Monitoring System Using a Ceiling-Attached Microwave Antenna. Medical and Biological Engineering and Computing, 44, 835-840. https://doi.org/10.1007/s11517-006-0091-8

10. Suzuki, S., Matsui, T., Kagawa, M., Asao, T. and Kotani, K. (2013) An Approach to a Non-Contact Vital Sign Monitoring Using Dual-Frequency Microwave Radars for Elderly Care. Journal of Biomedical Science and Engineering, 6, 704-711. https://doi.org/10.4236/jbise.2013.67086

11. Suzuki, S., Matsui, T., Imuta, H., Uenoyama, M., Yura, H., Ishihara, M. and Kawakami, M. (2008) A Novel Autonomic Activation Measurement Method for Stress Monitoring: Non-Contact Measurement of Heart Rate Variability Using a Compact Microwave Radar. Medical and Biological Engineering and Computing, 46, 709-714. https://doi.org/10.1007/s11517-007-0298-3

12. Droitcour, A.D. and Lubecke, O.B. (2016) Physiological Motion and Measurement. In: Lubecke, O.B., Lubecke, V.M., Droitcour, A.D., Park, B.K. and Singh, A., Eds., Doppler Radar Physiological Sensing, John Wiley \& Sons, Inc., Hoboken, 39-67. https://doi.org/10.1002/9781119078418.ch3

13. Golberg, M., Ruiz-Rivas, J., Polani, S., Beiderman, Y. and Zalevsky, Z. (2018) Large-Scale Clinical Validation of Noncontact and Continuous Extraction of Blood Pressure via Multipoint Defocused Photonic Imaging. Applied Optics, 57, B45. https://doi.org/10.1364/AO.57.000B45

14. Standards Committee of the IEEE Engineering in Medicine and Biology Society (2014) IEEE Standard for Wearable, Cuffless Blood Pressure Measuring Devices. IEEE-SA Standards Board. http://ieeexplore.ieee.org/stamp/stamp.jsp?tp=\&arnumber=6882122\&isnumber $=6882121 \% 0 \mathrm{~A}$

15. Kaplan, N.M., Victor, R.G. and Flynn, J.T. (2010) Kaplan's Clinical Hypertension. 10th Edition, Lippincott Williams \& Wilkins, Philadelphia.

16. Tijsseling, A.S. and Anderson, A. (2012) A. Isebree Moens and D.J. Korteweg: On the Speed of Propagation of Waves in Elastic Tubes. BHR Group 11 th International Conferences on Pressure Surges, Lisbon, 24-26 October 2012, 227-245.

17. Bramwell, J.C. and Hill, A.V. (1922) The Velocity of the Pulse Wave in Man. Proceedings of the Royal Society of London. Series B, 93, 298-306. https://doi.org/10.1098/rspb.1922.0022

18. Gosling, R.G. and Budge, M.M. (2003) Terminology for Describing the Elastic Behavior of Arteries. Hypertension, 41, 1180-1182. https://doi.org/10.1161/01.HYP.0000072271.36866.2A

19. Sun, C.K. (2013) Cardio-Ankle Vascular Index (CAVI) as an Indicator of Arterial Stiffness. Integrated Blood Pressure Control, 6, 27-38. https://doi.org/10.2147/IBPC.S34423 
20. Johar, R.S. and Smith, R.P. (2009) Assessing Gravimetric Estimation of Intraoperative Blood Loss. Journal of Gynecologic Surgery, 9, 151-154. https://doi.org/10.1089/gyn.1993.9.151

21. Ding, X., Yan, B.P., Zhang, Y.T., Liu, J., Zhao, N. and Tsang, H.K. (2017) Pulse Transit Time Based Continuous Cuffless Blood Pressure Estimation. A New Extension and a Comprehensive Evaluation. Scientific Reports, 7, Article No. 11554. https://doi.org/10.1038/s41598-017-11507-3

22. Geddes, L.A., Voelz, M.H., Babbs, C.F., Bourland, J.D. and Tacker, W.A. (1981) Pulse Transit Time as an Indicator of Arterial Blood Pressure. Psychophysiology, 18, 71-74. https://doi.org/10.1111/j.1469-8986.1981.tb01545.x

23. ISO 7250-1:2017 Basic Human Body Measurements for Technological Design—Part 1: Body Measurement Definitions and Landmarks. ISO/TC 159/SC 3 Anthropometry and Biomechanics.

24. Klabunde, R.E. (2011) Cardiovascular Physiology Concepts. 2nd Edition, Lippincott Williams \& Wilkins, Philadelphia.

25. Kaplan, A.D., O’Sullivan, J.A., Sirevaag, E.J., Lai, P.H. and Rohrbaugh, J.W. (2012) Hidden State Models for Noncontact Measurements of the Carotid Pulse Using a Laser Doppler Vibrometer. IEEE Transactions on Biomedical Engineering, 59, 744-753. https://doi.org/10.1109/TBME.2011.2179297

26. Smith, S.A., Salih, M.M. and Littler, W.A. (1987) Assessment of Beat to Beat Changes in Cardiac Output during the Valsalva Manoeuvre Using Electrical Bioimpedance Cardiography. Clinical Science, 72, 423-428. https://doi.org/10.1042/cs0720423

27. Risk, M., Berghoff, M. and Freeman, R. (2000) Characterization of the Valsalva Maneuver Using Wavelet Transform. Computers in Cardiology, 2000, 411-414. https://doi.org/10.1109/CIC.2000.898544

28. Van Vliet, B.N., McGuire, J., Chafe, L., Leonard, A., Joshi, A. and Montani, J.P. (2006) Phenotyping the Level of Blood Pressure by Telemetry in Mice. Clinical and Experimental Pharmacology and Physiology, 33, 1007-1015. https://doi.org/10.1111/j.1440-1681.2006.04479.x

29. Goncalves, A.C.C., Tank, J., Diedrich, A., Hilzendeger, A., Plehm, R., Bader, M., Luft, F.C., Jordan, J. and Gross, V. (2009) Diabetic Hypertensive Leptin Receptor-Deficient db/db Mice Develop Cardioregulatory Autonomic Dysfunction. Hypertension, 53, 387-392. https://doi.org/10.1161/HYPERTENSIONAHA.108.124776

30. Stewart, J.M., Medow, M.A., Bassett, B. and Montgomery, L.D. (2004) Effects of Thoracic Blood Volume on Valsalva Maneuver. American Journal of Physiology - Heart and Circulatory Physiology, 287, 798-804. https://doi.org/10.1152/ajpheart.01174.2003

31. Smith, G. and Boyle, M.J. (2009) The $10 \mathrm{~mL}$ Syringe Is Useful in Generating the Recommended Standard of 40 mmHg Intrathoracic Pressure for the Valsalva Manoeuvre. Emergency Medicine Australasia, 21, 449-454. https://doi.org/10.1111/j.1742-6723.2009.01228.x

32. Mukkamala, R., Hahn, J.O., Inan, O.T., Mestha, L.K., Kim, C.S., Toreyin, H. and Kyal, S. (2015) Toward Ubiquitous Blood Pressure Monitoring via Pulse Transit Time: Theory and Practice. IEEE Transactions on Biomedical Engineering, 62, 1879-1901. https://doi.org/10.1109/TBME.2015.2441951

33. Jacobsen, M., Dembek, T.A., Kobbe, G., Gaidzik, P.W. and Heinemann, L. (2020) Noninvasive Continuous Monitoring of Vital Signs with Wearables: Fit for Medical Use? Journal of Diabetes Science and Technology, 15, 34-43. https://doi.org/10.1177/1932296820904947

34. Suzuki, S., Matsui, T., Asao, T. and Kotani, K. (2012) An Investigation Using High-Precision CCD Laser Displacement Sensor to Measure Body Surface Motion Induced by Heartbeat. Journal of Biomedical Science and Engineering, 5, 672-677. https://doi.org/10.4236/jbise.2012.511084

35. Killip, T. and Kimball, J.T. (1967) Treatment of Myocardial Infarction in a Coronary Care Unit: A Two Year Experience with 250 Patients. The American Journal of Cardiology, 20, 457-464. 
https://doi.org/10.1016/0002-9149(67)90023-9

36. Khot, U.N., Jia, G., Moliterno, D.J., Lincoff, A.M., Khot, M.B., Harrington, R.A. and Topol, E.J. (2003) Prognostic Importance of Physical Examination for Heart Failure in Non-ST-Elevation Acute Coronary Syndromes. JAMA, 290, 2174-2181. https://doi.org/10.1001/jama.290.16.2174

37. Swan, H.J.C., Forrester, J.S., Diamond, G. and Parmley, W.W. (1972) Hemodynamic Spectrum of Myocardial Infarction and Cardiogenic Shock. Circulation, 45, 1097-1110. https://doi.org/10.1161/01.CIR.45.5.1097 